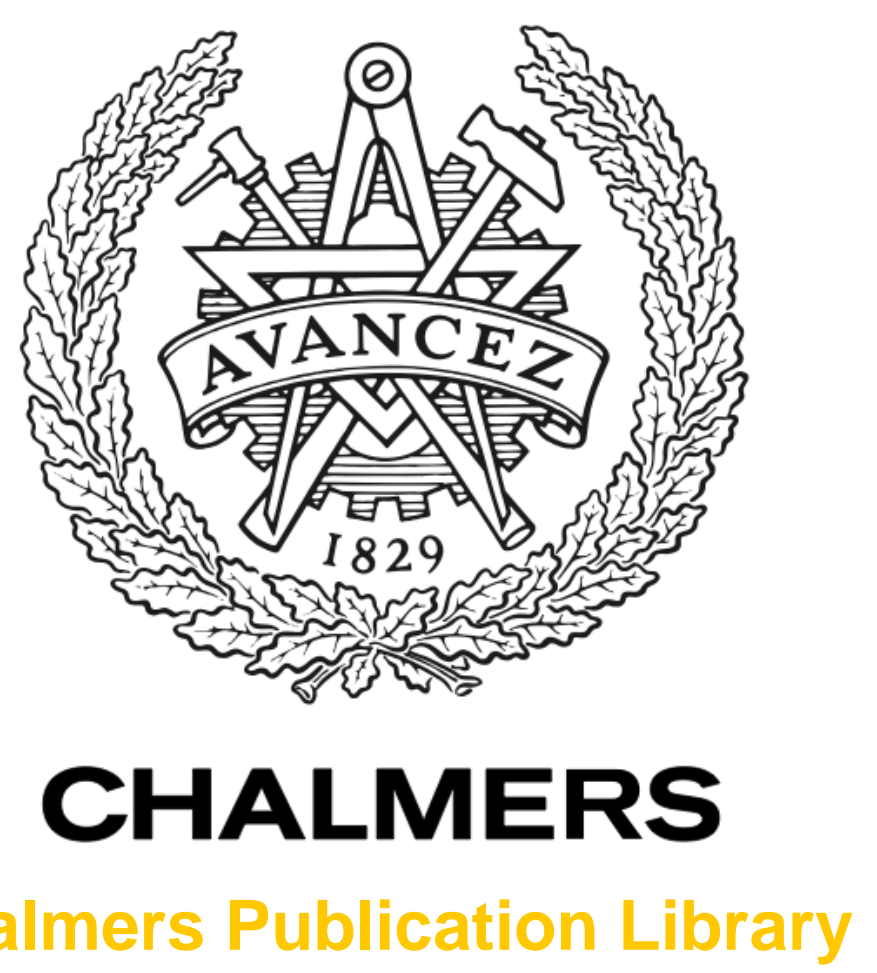

\title{
Superconducting weak bonds at grain boundaries in MgB2
}

This document has been downloaded from Chalmers Publication Library (CPL). It is the author's version of a work that was accepted for publication in:

JETP Letters (ISSN: 0021-3640)

Citation for the published paper:

Tarasov, M. ; Stepantsov, E. ; Naito, M. (2007) "Superconducting weak bonds at grain boundaries in MgB2". JETP Letters, vol. 105(3), pp. 636-641.

Downloaded from: http://publications.lib.chalmers.se/publication/63444

Notice: Changes introduced as a result of publishing processes such as copy-editing and formatting may not be reflected in this document. For a definitive version of this work, please refer to the published source. Please note that access to the published version might require a subscription. 


\title{
ELECTRONIC PROPERTIES OF SOLIDS
}

\section{Superconducting Weak Bonds at Grain Boundaries in $\mathrm{MgB}_{2}$}

\author{
M. A. Tarasov ${ }^{a}$, E. A. Stepantsov ${ }^{b}$ M. Naito ${ }^{c}$, A. Tsukada ${ }^{c}$, D. Winkler ${ }^{d}$, \\ A. S. Kalabukhov ${ }^{d, e}$, and M. Yu. Kupriyanov ${ }^{e}$ \\ ${ }^{a}$ Institute of Radio Engineering and Electronics, Russian Academy of Sciences, Moscow, 125009 Russia \\ ${ }^{b}$ Institute of Crystallography, Russian Academy of Sciences, Moscow, 119333 Russia \\ ${ }^{c}$ Department of Applied Physics, Tokyo University of Agriculture and Technology, 2-24-16, \\ Naka-cho, Kogenei, Tokyo, 184-8588 Japan \\ ${ }^{d}$ Department of Microtechnology and Nanoscience, Chalmers University of Technology, Göteborg, 41296 Sweden \\ ${ }^{e}$ Research Institute of Nuclear Physics, Moscow State University, Moscow, 119992 Russia \\ e-mail:tarasov@hitech.cplire.ru \\ Received January 5, 2007
}

\begin{abstract}
The possibility of preparing bicrystalline Josephson junctions and bolometers based on superconducting $\mathrm{MgB}_{2}$ on specially prepared bicrystalline $\mathrm{MgO}$ substrates is investigated. Microbridges $0.85-6.00 \mu \mathrm{m}$ in width, intersecting the bicrystalline interface, are formed in epitaxial bicrystalline $\mathrm{MgB}_{2}$ films grown on these substrates. It is found that annealing of bicrystalline samples in oxygen leads to a systematic decrease in the critical current, an increase in the temperature width of the superconducting transition region, and to an improvement of the current-voltage (IV) characteristic, which becomes close in shape to the $I V$ characteristic of a Josephson junction. The response of such a junction to radiation at a frequency of $110 \mathrm{GHz}$ with an amplitude attaining $0.5 \mathrm{mV}$ is measured.
\end{abstract}

PACS numbers: 74.45.+c, 74.50.+r

DOI: $10.1134 / \mathrm{S} 106377610709021 \mathrm{X}$

\section{INTRODUCTION}

The superconducting transition temperature in magnesium diboride $\left(\mathrm{MgB}_{2}\right)$ is $39 \mathrm{~K}$, which is the highest value for compounds that do not contain copper oxide layers. This substance is a classical superconductor obeying the Bardeen-Cooper-Schrieffer (BCS) theory with a relatively large coherence length $\xi_{c}(0) \approx 2.5 \mathrm{~nm}$ in the direction of the $c$ axis and $\xi_{a b}(0) \approx 10 \mathrm{~nm}$ in the $a b$ plane. The corresponding values in a $\mathrm{YBaCuO}$ superconductor are almost an order of magnitude lower $\xi_{c}(0) \approx 0.2-0.5 \mathrm{~nm}$ and $\left.\xi_{a b}(0) \approx 1.5-3.0 \mathrm{~nm}\right)$. This difference turns out to be an important factor for manufacturing homogeneous and reproducible Josephson junctions.

The considerable interest in the problem of preparing $\mathrm{MgB}_{2}$-based Josephson structures is due to the potentially high values of their characteristic voltage. The energy gap in these structures is $\Delta_{\sigma}(0) \approx 2.09 k T_{c}=$ $7.09 \mathrm{mV}$ in the $\sigma$ band and $\Delta_{\pi}(0) \approx 0.8 k T_{c}=2.7 \mathrm{mV}$ in the $\pi$ band [1]. These values of the gap correspond to characteristic frequencies of 3.5 and $1.3 \mathrm{THz}$, which are substantially higher than the frequency limitations of $700 \mathrm{GHz}$ for niobium junctions.

Until recently, $\mathrm{MgB}_{2}$-based Josephson structures were mainly prepared using methods that did not permit reproducible control of the parameters of junctions or the regions of weak bond localization. Such structures include point contacts, junctions at defects emerg- ing under the action of mechanical stresses (which will be referred to as break junctions), and structures of a type of bridge with a varying thickness, in which a weak spot was produced either directly by ion implantation, or at random defects formed upon a decrease in the thickness of a part of the initial film [2-11].

In more controllable structures, one electrode was, as a rule, a low-temperature superconductor $(\mathrm{Nb}, \mathrm{Al}$, $\mathrm{NbN}$ ) [12-16]. The properties of $\mathrm{MgB}_{2} / \mathrm{I} / \mathrm{MgB}_{2}$ tunnel junctions, in which $\mathrm{AlO}_{x}[17], \mathrm{AlN}[18,19]$, and $\mathrm{MgO}$ $[20,21]$ were used as the insulating (I) interlayer, have been reported relatively recently.

Unfortunately, the parameters of Josephson junctions reported in these publications were far from optimal. This served as a stimulus for searching for different methods for preparing $\mathrm{MgB}_{2}$-based Josephson structures for high-frequency application.

We proposed that a better weak link be formed by growing a $\mathrm{MgB}_{2}$ film on a bicrystalline substrate of magnesium oxide oriented in the (111) plane ( $\mathrm{MgO}(111))$. Mismatching in the lattice constants of $\mathrm{MgO}$ in the $\langle 110\rangle$ direction $(a=0.298 \mathrm{~nm})$ and of the $\mathrm{MgB}_{2}(001)$ film in the same direction $(a=0.308 \mathrm{~nm})$ is just $3.4 \%$. For this reason, high-quality $\mathrm{MgB}_{2}$ epitaxial films can be grown on such a bicrystalline substrate and, as a consequence, an artificial grain boundary intersecting the entire film thickness can emerge. Subsequent annealing of such structures in oxygen must be accompanied by 
its intense diffusion along the artificially produced interface between the films with the formation of an insulating $\mathrm{MgO}$ or $\mathrm{B}_{2} \mathrm{O}_{3}$ layer. Boundaries of the 1-3 nm thick $\mathrm{B}_{2} \mathrm{O}_{3}$ layer between $\mathrm{MgB}_{2}$ grains were observed with the help of a transmission electron microscope in [22]. The interface can also be metallic with the composition $\mathrm{MgB}_{2}$ [23] or amorphous with the same composition and a thickness of 5-20 nm, as was observed in [24]. The boundaries of these three types can be used for preparing Josephson junctions of the superconductor-insulator-superconductor (SIS) or superconductor-normal metal-superconductor (SNS) structure.

\section{SAMPLE PREPARATION TECHNIQUE}

$\mathrm{MgO}$ (111) bicrystalline substrates were prepared by solid-phase splicing [25]. For this purpose, two $\mathrm{MgO}$ single crystals were brought in contact over the $\left[(110)-13^{\circ}\right]$ and $\left[(110)+13^{\circ}\right]$ planes for one type of bicrystals and over the $\left[(112)-13^{\circ}\right]$ and $\left[(112)+13^{\circ}\right]$ planes for another type of bicrystals. In both configurations, the (111) planes of the blocks being spliced were parallel. The sealing was carried out under ultrahigh vacuum, heating, and compression. Bicrystals were cut into $0.5-\mathrm{mm}$-thick substrates parallel to the (111) plane and polished. In this way, bicrystalline substrates with the (111) orientation were prepared with an artificial grain boundary perpendicular to the surface and with symmetric rotation of the crystal lattices through $13^{\circ}$ in opposite directions from the boundary. The boundary formed an angle of $13^{\circ}$ with the $\langle 112\rangle$ axes in the first type of substrates and with the $\langle 110\rangle$ axes in the second type of substrates. Monocrystalline substrates with the (111) orientation were also prepared for comparison.

$\mathrm{MgB}_{2}$ films were grown by thermal evaporation in ultrahigh vacuum of the specially designed original evaporation unit (residual gas pressure, $5 \times 10^{-10}$ Torr), made in the form of two (pure magnesium and pure boron) targets using various electron guns. The evaporation rate for each element was monitored using electron impact emission spectrometry (EIES). The temperature of the substrate was $280^{\circ} \mathrm{C}$ and the film growth rate was $0.38 \mathrm{~nm} / \mathrm{s}$. To compensate for magnesium losses due to resputtering, the magnesium evaporation rate relative to boron was three times as high as the nominal value. Peculiarities of the film growth were described in detail in [26].

Heterostructures consisting of a $\mathrm{MgO}$ (111) substrate and $\mathrm{a}_{\mathrm{MgB}}$ (001) film were analyzed in an $\mathrm{X}$-pert Philips four-circle X-ray diffractometer. We chose the (311)-type planes as skew X-rays reflection planes for the MgO (111) substrate. The (112) reflecting planes were chosen for the films. The results of measurement of X-ray diffraction curves during rotation of the sample about the normal to the surface $(\varphi$ scan) are presented in Fig. 1. It can be seen from Fig. 1a that the peaks of reflection from the (112) planes are displaced by $60^{\circ}$ relative to the reflections

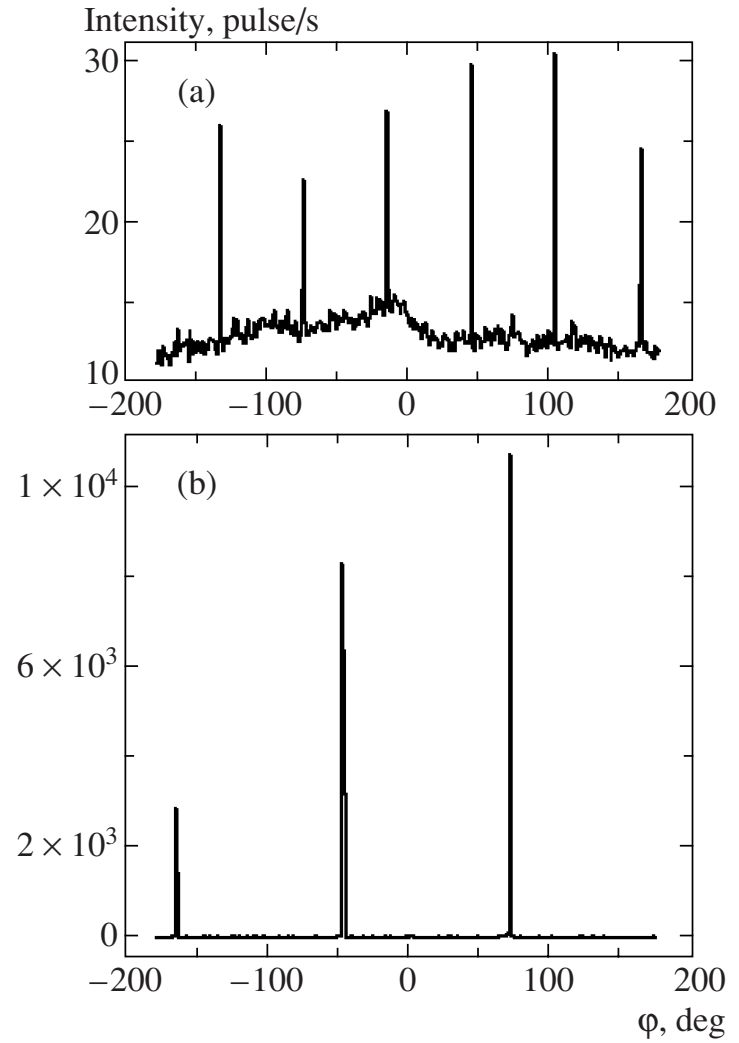

Fig. 1. Curves recorded in X-ray diffractometric scanning of the $\mathrm{MgB}_{2}$ (001) film and a substrate made of a $\mathrm{MgO}$ (111) crystal, on which the film was grown during their rotation about the $\langle 001\rangle$ axis of the film and for the established Bragg position of the source-detector system: (a) for the film plane of the (112) type and (b) for the substrate plane of the (311) type.

from the (311) planes of the substrate in Fig. 1b. This means that the crystal lattice in $\mathrm{MgB}_{2}$ (001) films is epitaxially coupled to the lattice of the $\mathrm{MgO}$ (111) substrate. The $\langle 110\rangle$ direction on the interface plane in the film is parallel to the $\langle 110\rangle$ direction of the substrate.

Bridges $6 \mu \mathrm{m}$ in length and $6,4,1.5$, and $0.85 \mu \mathrm{m}$ in thickness, which intersected the artificial grain boundary, were formed in the $\mathrm{MgB}_{2}$ film by dry ionic etching. Each bridge was connected with a planar antenna. Figure 2 shows an optical image of such an integrated structure with a log-periodic antenna and a bicrystalline microbridge at the center.

\section{EXPERIMENTAL RESULTS AND DISCUSSION}

The current-voltage (IV) characteristics of the samples measured immediately after their preparation were characterized by critical currents from 1.5 to $10 \mathrm{~mA}$ and a normal resistance from 20 to $60 \Omega$. Then several annealing cycles were performed for $1 \mathrm{~h}$ in an oxygen atmosphere under a pressure of $800 \mathrm{mbar}$ at temperatures of $100,200,300,450$, and $600^{\circ} \mathrm{C}$. Annealing of films on bicrystalline substrates in oxygen led to a sys- 


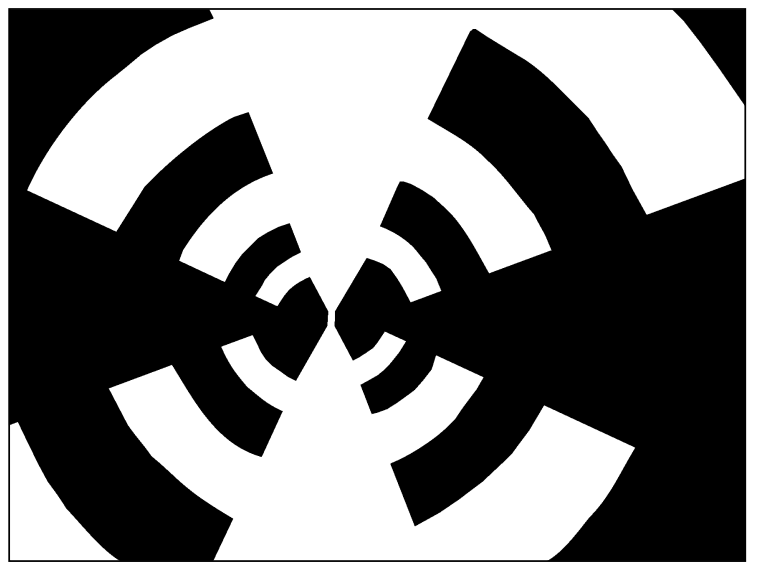

Fig. 2. Optical image of an integrated structure containing a log-periodic antenna and $\mathrm{agB}_{2}$ microbridge intersecting the bicrystalline boundary at the center.

tematic decrease in the critical current, an expansion of the temperature range of the transition, and a transformation of the shape of $I V$ curves.
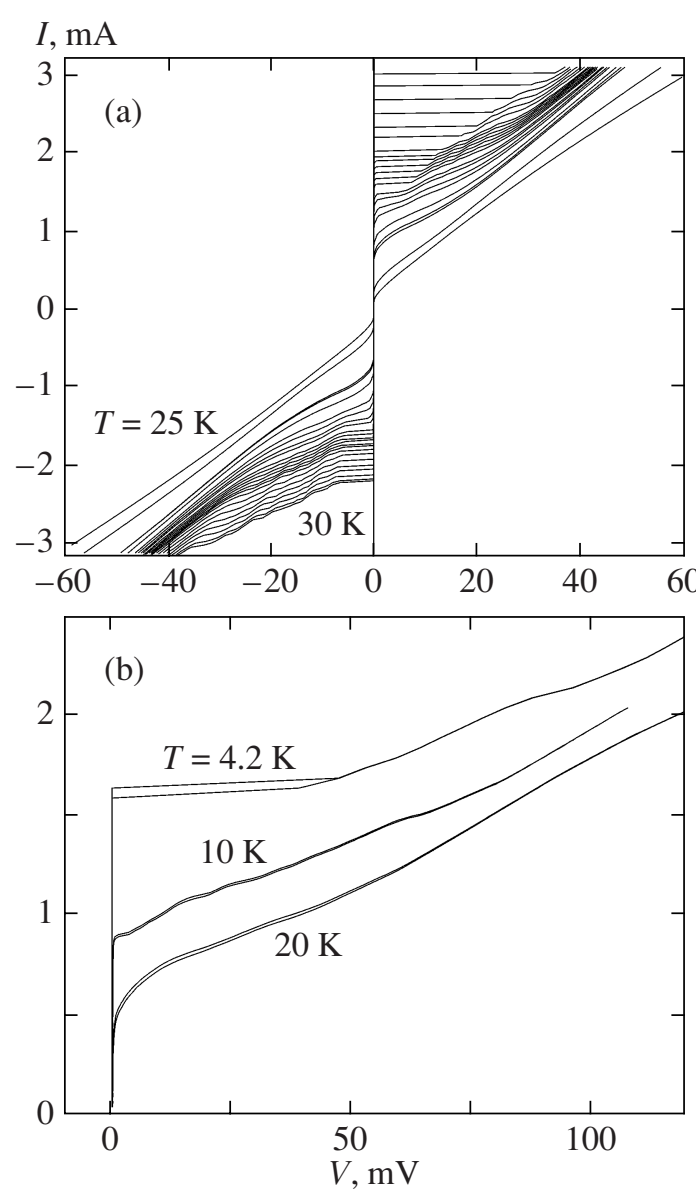

Figure 3 shows the $I V$ characteristics of the structures obtained as a result of annealing at $450^{\circ} \mathrm{C}$ of $0.9-\mu \mathrm{m}$-wide bridges prepared on a $\langle 110\rangle$ bicrystalline substrate and on a monocrystalline substrate. Measurements were performed in the temperature interval 4.2-30 K. Figure $3 \mathrm{c}$ shows the IV characteristics in various magnetic fields up to $3.5 \mathrm{~T}$ at a temperature of $28 \mathrm{~K}$. It can be seen that, as a result of annealing, the $I V$ curves acquire the shape typical of shunted Josephson junctions. Upon a decrease in temperature, the IV curves acquire a hysteresis. Annealing of a $\langle 112\rangle$ bicrystalline sample at $600^{\circ} \mathrm{C}$ led to complete vanishing of the critical current and to an increase in the resistance up to $1 \mathrm{k} \Omega$ for the broadest $(6-\mu \mathrm{m})$ junction and to infinity for the remaining junctions.

Figure 4 shows the temperature dependences of the critical current for $0.9-\mu \mathrm{m}$-thick bridges on bicrystalline and monocrystalline substrates after annealing for $1 \mathrm{~h}$ at $600^{\circ} \mathrm{C}$. It can be seen that the absolute values of the critical currents and their temperature dependences are different. This means that oxidation of the artificially prepared interface in $\mathrm{MgB}_{2}$ on a bicrystalline substrate indeed occurs at a higher rate and leads to the
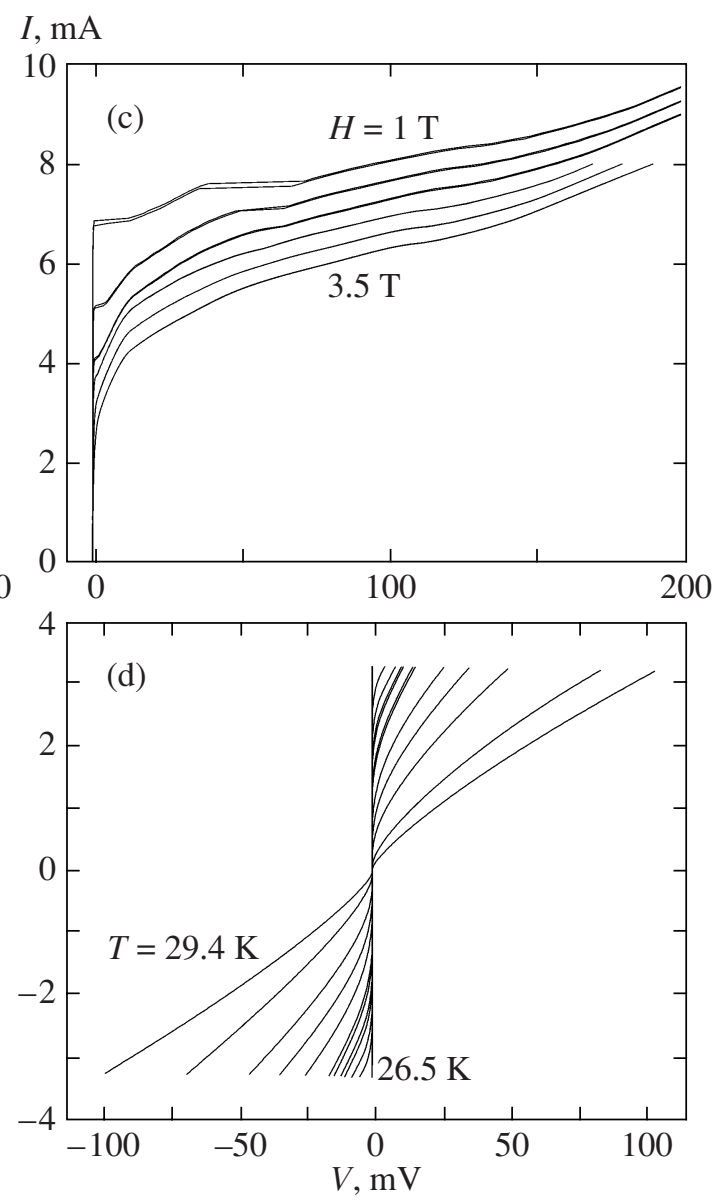

Fig. 3. $I V$ characteristics for a $\mathrm{MgB}_{2}\langle 110\rangle$ bicrystalline bridge $0.9 \mu \mathrm{m}$ in width, annealed at $450^{\circ} \mathrm{C}$, which were measured at temperatures of (a) 25-30, (b) 20, 10, 4.2, and (c) $28 \mathrm{~K}$ in magnetic fields of 1, 1.5, 2, 2.5, 3, and $3.5 \mathrm{~T}$, as well as (d) $I V$ curves for annealed monocrystalline bridge of thickness $0.9 \mu \mathrm{m}$ at $26.5-29.4 \mathrm{~K}$. 
emergence of a weak spot in the film in which the critical current is lower than in the remaining parts of the film.

In Fig. 5, the temperature dependences $I_{c}(T)$ of the critical current are compared with the available theoretical dependences calculated for tunnel SIS structures [1] and two-barrier SINIS structures [27] in the case of tunneling along the $c$ axis. The same figure shows the experimental data obtained in structures with weak coupling at natural boundaries in $\mathrm{MgB}_{2}$ [10] and in break junctions $[4,5,8]$ in the structures formed by ion implantation [3], etching by a focused ion beam [2], and in tunnel junctions of $\mathrm{MgB}_{2} / \mathrm{AlO}_{x} / \mathrm{MgB}_{2}$ [17] and $\mathrm{MgB}_{2} / \mathrm{AlN} / \mathrm{MgB}_{2}[18,19]$.

It can be seen that the available experimental data can be conditionally divided into three groups. The first group includes the results obtained in [2], where the temperature dependence has a positive curvature and the slope of the curve in the vicinity of the critical temperature is noticeably higher than for the remaining aggregate of data. In our opinion, this is due to the fact that the dynamic state in the structures studied in [2] was formed due to depinning of Abrikosov vortices rather than as a result of breaking of the Josephson coupling.

The second group of results was obtained in break junctions $[4,5,8]$ and in $\mathrm{MgB}_{2} / \mathrm{MgO} / \mathrm{MgB}_{2}$ junctions $[20,21]$. These data are characterized by an excessively large (as compared to theoretical curves) negative curvature of the $I_{c}(T)$ curves. Such a behavior of the curves is apparently associated with the nonuniformity of the superconducting properties over the width of the junction. In this case, the decrease in temperature must be accompanied by not only an increase in the modulus of the order parameter of the electrodes, but also by the inclusion of an additional critical current transport channel. The latter circumstance is precisely responsible for a higher rate of increase of $I_{c}$ and a decrease in temperature.

The third group of data, including the results obtained in $[3,10,18,19]$, as well as our results, exhibits a satisfactory agreement between the experimental dependences $I_{c}(T)$ and the shape of the curve calculated for two-barrier SINIS structures in the limit of small values of the effective suppression parameter. The negative curvature of this curve is a consequence of the two-band nature of the $\mathrm{MgB}_{2}$ compound. It is absent in analogous structures with one-band superconductors [28] as well as $\mathrm{MgB}_{2} / \mathrm{I} / \mathrm{MgB}_{2}$ tunnel junctions with tunneling in the $a b$ plane [1]. The latter circumstance is not accidental. It was shown in [1] that even an insignificant disorientation (exceeding $0.6^{\circ}$ ) of crystallographic directions $c$ of contacting $\mathrm{MgB}_{2}$ blocks leads to the same result of tunneling in the $a b$ plane as tunneling in the $c$ direction. It should also be noted that the satisfactory agreement with the shape of the curve calculated for two-barrier SINIS structures does not mean that we are dealing precisely with the junction formed by a nor-

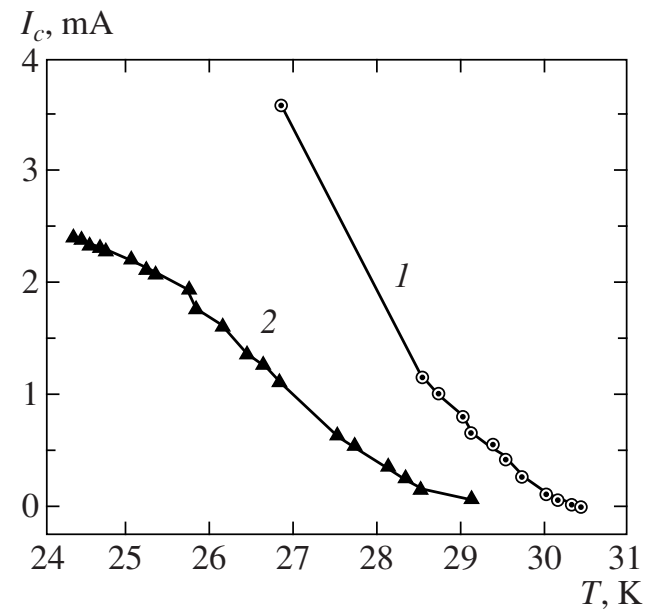

Fig. 4. Temperature dependences of the critical current of a $0.9-\mu \mathrm{m}$-wide bridge on a monocrystalline (1) and bicrystalline (2) substrates after annealing.

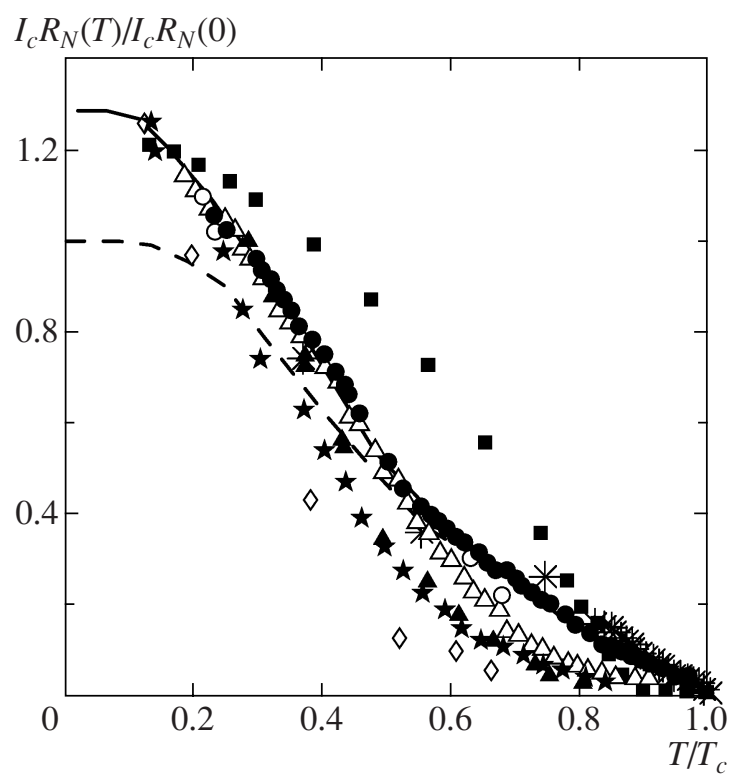

Fig. 5. Comparison of our temperature dependence of critical current (crosses) with the available theoretical dependences calculated for tunnel SIS structures [1] (dashed curve) and two-barrier SINIS structures [27] (solid curve) for tunneling along the $c$ axis with the results obtained by other authors: $\boldsymbol{\square}[2], \triangle[3], \boldsymbol{\Delta}[5], \bigcirc[10], \bullet[14,18,19], \square$ $[8], \diamond[17]$, and $\star[4]$.

mal metal sandwiched between two two-band superconductors. The same result can be obtained if we have a tunnel junction with localized states located at the center of a tunnel layer with an energy uniformly distributed in the vicinity of the Fermi energy [28]. Comparison precisely with the shape of the $I_{c}(T)$ curve is necessitated by the following factors. The spatial distributions of the superconducting and normal current components in the structures considered here, as well as bicrystalline HTSC junctions, do not coincide as a 

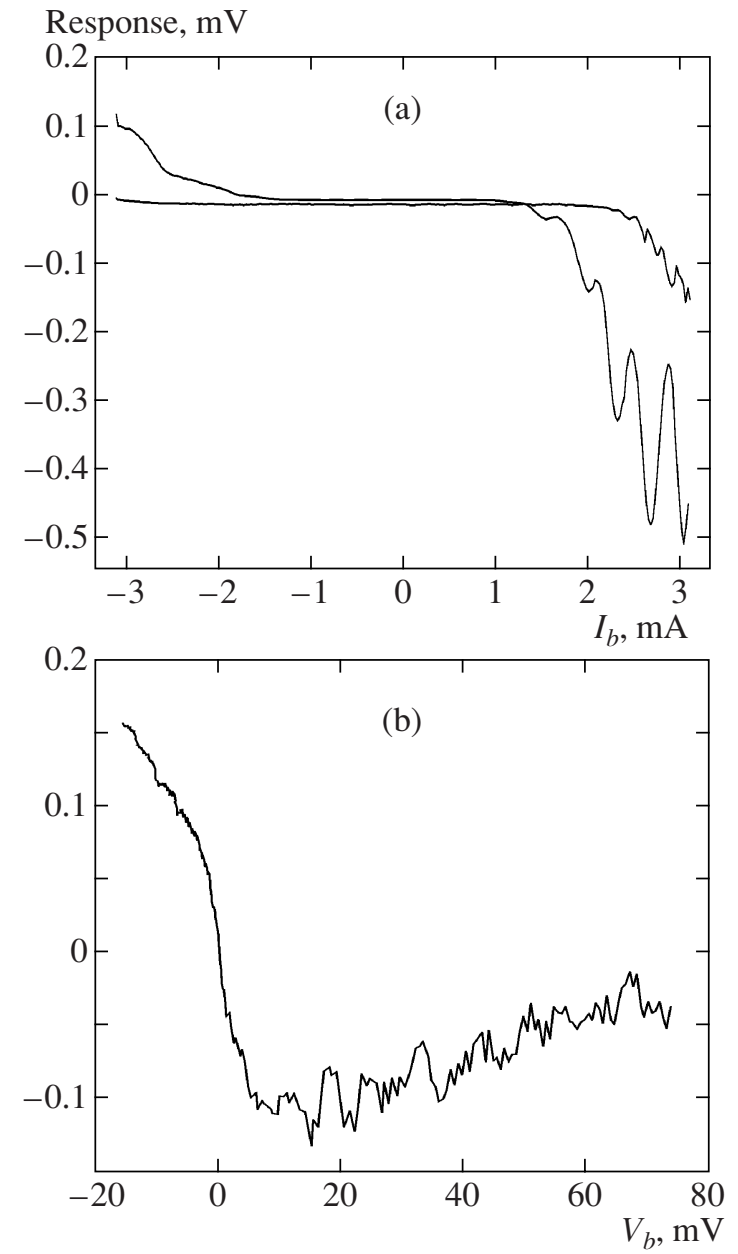

Fig. 6. Voltage response to radiation at a frequency of $110 \mathrm{GHz}$ as a function of (a) the bias current for two different signal levels and (b) the bias voltage.

rule. This leads to the emergence of a shunting resistance and suppression of the values of characteristic voltage [29], which complicates the comparison of absolute experimental values of $I_{c} R_{N}$ with the theory ( $R_{N}$ is the resistance of the normal metal).

Thus, we have shown that oxidation along the bicrystalline boundary makes it possible to form controllable weak bonds in $\mathrm{MgB}_{2}$ in the $a b$ plane. We also measured the voltage response for such junctions irradiated at a frequency of $110 \mathrm{GHz}$. Radiation emitted by an impact avalanche transit-time (IMPATT) diode via a scalar horn, attenuators, and an optical window with cold filters was fed to the cold stage of the cryostat. The samples were placed on the plane surface of an extended hyperhemispherical sapphire lens. Figure 6 shows the dependences of the response on the bias current and voltage. The peaks of the response correspond to the positions of the differential resistance peaks. Shapiro steps were not observed in explicit form, which can be due to the nonuniform distribution of the current over the bridge thickness. In a wider range of bias voltages, the response gradually decreases, which may be due to overheating by the bias current. The amplitude of the response attained $0.5 \mathrm{mV}$, which makes it possible to use junctions of this type as bolometric receivers at a temperature of about $30 \mathrm{~K}$.

\section{CONCLUSIONS}

We prepared $\mathrm{MgB}_{2}$ bridges $0.85-6.00 \mu \mathrm{m}$ in width on monocrystalline and bicrystalline $\mathrm{MgO}$ (111) substrates of two types: $13^{\circ} / 13^{\circ}\langle 110\rangle$ and $13^{\circ} / 13^{\circ}\langle 112\rangle$. The 100 -nm-thick $\mathrm{MgB}_{2}$ films had a critical temperature of $T_{\mathrm{c}}=34 \mathrm{~K}$. The critical currents of $0.85-\mu \mathrm{m}$-thick samples immediately after their preparation were 1.5-10 mA for normal resistances of 20-60 $\Omega$. Samples were annealed in oxygen for $1 \mathrm{~h}$ at 100, 200,300, 450, and $600^{\circ} \mathrm{C}$. Annealing of films on bicrystalline substrates led to a systematic decrease in critical current, completely suppressed its hysteresis, extended the temperature range of the junctions, and improved the shape of $I V$ characteristics, which resembled in this case the $I V$ curves of Josephson junctions. Bridges on a monocrystalline substrate did not exhibit any noticeable changes. Thus, we proved that oxidation along the bicrystalline boundary makes it possible to form controllable weak bonds in $\mathrm{MgB}_{2}$ bridges. The absence of explicit oscillations in the dependences on the magnetic film and microwave radiation power may be due to the nonuniformity of the current distribution of the current along the bridge boundary. We also measured the voltage response in such junctions upon irradiation at a frequency of $110 \mathrm{GHz}$ with an amplitude of up to $0.5 \mathrm{mV}$, which enables us to consider the possibility of using such junctions as bolometric receivers at a temperature of about $30 \mathrm{~K}$.

\section{ACKNOWLEDGMENTS}

This study was supported by VR and SI Swedish agencies, the Russian Foundation for Basic Research (project no. 05-02-19650), and the Federal Agency for Science and Innovations (grant no. 02.513.11.3157).

\section{REFERENCES}

1. A. Brinkman, A. A. Golubov, H. Rogalla, et al., Phys. Rev. B 65, 180517 (2002).

2. A. Brinkman, D. Veldhuis, D. Mijatovic, et al., Appl. Phys. Lett. 79, 2420 (2001).

3. G. Burnell, D. J. Kang, H. N. Lee, et al., Appl. Phys. Lett. 79, 3464 (2001).

4. Y. Zhang, D. Kinion, J. Chen, et al., Appl. Phys. Lett. 79, 3995 (2001).

5. R. S. Gonnelli, A. Calzolari, D. Daghero, et al., Phys. Rev. Lett. 87, 097001 (2001).

6. Z. Z. Li, Y. Xuan, H. J. Tao, et al., Physica C (Amsterdam) 370, 1 (2002).

7. H. Schmidt, J. F. Zasadzinski, K. E. Gray, and D. G. Hinks, Phys. Rev. Lett. 88, 127002 (2002). 
8. H.-J. Tao, Z.-Z. Li, Y. Xuan, et al., Physica C (Amsterdam) 386, 569 (2003).

9. G. Burnell, D.-J. Kang, D. A. Ansell, et al., Supercond. Sci. Technol. 16, 254 (2003).

10. A. Malisa, M. Valkeapää, L. Johansson, and Z. Ivanov, Supercond. Sci. Technol. 17, S345 (2004).

11. N. Khare, D. P. Singh, A. K. Gupta, et al., Supercond. Sci. Technol. 17, 1372 (2004).

12. G. Carapella, N. Martucciello, G. Costabile, et al., Appl. Phys. Lett. 80, 2949 (2002).

13. A. Saito, A. Kawakami, H. Shimakage, et al., J. Appl. Phys. 92, 7369 (2002).

14. H. Shimakage, K. Tsujimoto, Z. Wang, and M. Tonouchi, Supercond. Sci. Technol. 17, 1376 (2004).

15. F. Giubileo, M. Aprili, F. Bobba, et al., Phys. Rev. B 72, 174518 (2005).

16. M. van Zalk, A. Brinkman, A. A. Golubov, et al., Supercond. Sci. Technol. 19, S226 (2006).

17. K. Ueda, S. Saito, K. Semba, and T. Makimoto, Appl. Phys. Lett. 86, 172502 (2005).

18. H. Shimakage, K. Tsujimoto, Z. Wang, and M. Tonouchi, Appl. Phys. Lett. 86, 072512 (2005).
19. H. Shimakage and Z. Wang, Supercond. Sci. Technol. 19, S182 (2006).

20. D. Mijatovic, A. Brinkman, I. Oomen, et al., Appl. Phys. Lett. 80, 2141 (2002).

21. P. Orgiani, Y. Cui, A. V. Pogrebnyakov, et al., IEEE Trans. Appl. Supercond. 15, 228 (2005).

22. R. F. Klie, J. C. Idrobo, N. D. Browning, et al., Appl. Phys. Lett. 79, 1837 (2001).

23. J. Q. Li, L. Li, Y. Q. Zhou, et al., Chin. Phys. Lett. 18, 680 (2001).

24. H. Narayan, S. B. Samanta, A. Gupta, et al., Physica C (Amsterdam) 377, 1 (2002).

25. E. A. Stepantsov, Byull. Izobret., No. 36, 77 (1984).

26. K. Ueda and M. Naito, J. Appl. Phys. 93, 2113 (2003).

27. A. Brinkman, A. A. Golubov, and M. Yu. Kupriyanov, Phys. Rev. B 69, 214407 (2004).

28. A. A. Golubov, M. Yu. Kupriyanov, and E. Il'ichev, Rev. Mod. Phys. 77, 411 (2004).

29. H. Hilgenkamp and J. Mannhart, Rev. Mod. Phys. 74, 485 (2002).

Translated by N. Wadhwa 